\title{
Computing turbulent far-wake development behind a wind turbine with and without swirl
}

\author{
Yingying $\mathrm{Hu}^{1}$, Siva Parameswaran*1, Jiannan $\operatorname{Tan}^{1}$, Suranga Dharmarathne ${ }^{1}$, \\ Neha Marathe ${ }^{2}$, Zixi Chen ${ }^{1}$, Ronald Grife ${ }^{3}$ and Andrew Swift ${ }^{2}$ \\ ${ }^{1}$ Department of Mechanical Engineering, Texas Tech University, Lubbock, Texas, USA \\ ${ }^{2}$ Wind Science and Engineering Research Center, Department of Civil Engineering, Texas Tech University, \\ Lubbock, Texas, USA \\ ${ }^{3}$ Vestas Technology R\&D Americas, Inc., USA \\ (Received September 5, 2010, Revised January 12, 2011, Accepted May 25, 2011)
}

\begin{abstract}
Modeling swirling wakes is of considerable interest to wind farm designers. The present work is an attempt to develop a computational tool to understand free, far-wake development behind a single rotating wind turbine. Besides the standard momentum and continuity equations from the boundary layer theory in two dimensions, an additional equation for the conservation of angular momentum is introduced to study axisymmetric swirl effects on wake growth. Turbulence is simulated with two options: the standard $k-\varepsilon$ model and the Reynolds Stress transport model. A finite volume method is used to discretize the governing equations for mean flow and turbulence quantities. A marching algorithm of expanding grids is employed to enclose the growing far-wake and to solve the equations implicitly at every axial step. Axisymmetric far-wakes with/without swirl are studied at different Reynolds numbers and swirl numbers. Wake characteristics such as wake width, half radius, velocity profiles and pressure profiles are computed. Compared with the results obtained under similar flow conditions using the computational software, FLUENT, this far-wake model shows simplicity with acceptable accuracy, covering large wake regions in far-wake study.
\end{abstract}

Keywords: far wake; swirl; boundary layer; self-similarity; $k-\varepsilon$ model; Reynolds Stress transport model.

\section{Introduction}

Many efforts have been made to develop renewable energy sources. Wind power is one of the most cost-effective ways to produce renewable energy (Herbert et al. 2007). Wind turbines are one of the oldest devices to harness the energy carried by wind. However, they cause clouds and energy inefficiency in their wakes. Wind turbines may lose $20 \%$ or $30 \%$ of their power due to wake effects (Mechali et al. 2006, Herbert et al. 2007). Aerodynamics of wind turbines is yet to be fully understood to the extent to which it can predict the power output reliably (Hansen et al. 2006). The chaotic behavior of the inflow wind makes the analysis even more complex.

In 2003, Vermeer et al. reviewed experimental and numerical studies on wind turbine aerodynamics in both near- and far-wakes. In 2009, Sanderse especially reviewed numerical

\footnotetext{
* Corresponding author, Dr., E-mail: siva.parameswaran@ttu.edu
} 
modeling of wakes. Recently, Sumner et al. (2010) also reviewed the development of computational fluid dynamics (CFD) in wind energy study. In the numerical study of near-wakes, the CFD methods based on the Navier-Stokes equations or vortex equations can yield accurate results. In the numerical study of far-wakes where the effects of turbine geometry are neglected, CFD methods are often computationally expensively due to the unsteadiness and turbulence of the wakes and domain meshing (Sanderse 2009, Sumner et al. 2010). For far-wake models with computational economy, assumptions are usually made to avoid numerical difficulty of large scale effects. For instance, the kinematic models are based on self-similar velocity deficit profiles (Lissaman 1979) or Gaussian profiles (Vermeulen 1980); the UPMWAKE code considers the boundary layer effects (Crespo et al. 1985, 1989). Compared with near-wakes behind wind turbines in a wind farm, wake interaction is a distinguished feature in far-wakes, and wake width needs to be predicted. Although there have been several models that study wake interaction through direct numerical simulation or assumption of linear superposition (Fletcher et al. 2009, Sanderse 2009, Vermeer et al. 2003), there are few studies on wake width prediction. The linear increase of the wake width with the downstream distance is an adopted assumption (Katic et al. 1986). In addition, swirl flow is investigated less in far-wakes than in near-wakes (Leschziner et al. 1984, Lilley 1973, Sanderse 2009, Vermeer et al. 2003).

To further understand the behavior of the far-wake behind a wind turbine with/without swirl (especially wake width prediction), we are to put forth a numerical model of the far-wake development using the boundary layer theory and self-similarity assumption. Two turbulence options, the standard $k-\varepsilon$ and Reynolds Stress (RS) transport models, are used to solve turbulent quantities. The computational code, EXPRESS (originally written for two-dimensional turbulent boundary layer flows), will be revised to perform the simulations. The CFD software package, FLUENT (ANSYS Inc., USA), is also used to perform similar calculations under the same boundary conditions for comparison purpose. Results about wake width, velocity profiles, pressure profiles, and added turbulence intensity will be discussed in the far-wake with/without swirl. The efficiency of the far-wake model will also be discussed.

\section{Basic theory}

\subsection{Equations governing mean flow}

Assume that flow in the far-wake is free, axisymmetric, and incompressible (Lilley 1973, Sanderse 2009). According to the boundary layer theory, the continuity equation and the momentum equations can be simplified as (Hogg et al. 1989, Lilley 1973)

$$
\begin{gathered}
\frac{\partial \rho U}{\partial x}+\frac{1}{r} \frac{\partial r \rho V}{\partial r}=0 \\
\frac{\partial \rho U^{2}}{\partial x}+\frac{1}{r} \frac{\partial r \rho U V}{\partial r}=\frac{1}{r} \frac{\partial}{\partial r}\left(r \mu \frac{\partial U}{\partial r}\right)-\frac{\partial p}{\partial x}-\frac{\partial r \rho \overline{u v}}{\partial r} \\
\frac{\partial \rho U r W}{\partial x}+\frac{\partial r \rho V r W}{\partial r}=\frac{\partial}{\partial r}\left(r \mu \frac{\partial r W}{\partial r}\right)-\frac{2 \mu}{r} \frac{\partial r W}{\partial r}-r \frac{\partial \rho \overline{v w}}{\partial r}-2 \rho \overline{v w}
\end{gathered}
$$




$$
\frac{\rho W^{2}}{r}=\frac{\partial p}{\partial r}
$$

in which $U, V$ and $W$ are mean velocity components in the axial $(x)$, radial $(r)$ and swirl (tangential $\theta$ ) directions, $p$ is pressure, $-\rho \overline{u v}$ and $-\rho \overline{v w}$ are two components of Reynolds stresses, $\rho$ is air density of $1.2 \mathrm{~kg} / \mathrm{m}^{3}$, and $\mu$ is viscosity of air, $1.8 \times 10^{-5} \mathrm{~Pa} \cdot \mathrm{s}$.

\section{2. $k-\varepsilon$ and Reynolds Stress transport models}

In the standard $k-\varepsilon$ model, the equations for the turbulence kinetic energy $k$ and its viscous dissipation rate $\varepsilon$ are (EXPRESS)

$$
\begin{gathered}
\frac{\partial \rho U k}{\partial x}+\frac{1}{r} \frac{\partial r \rho V k}{\partial r}=\frac{1}{r} \frac{\partial}{\partial r}\left[r\left(\mu+\frac{v_{t}}{\sigma_{k}}\right) \frac{\partial k}{\partial r}\right]+P-\varepsilon \\
\frac{\partial \rho U \varepsilon}{\partial x}+\frac{1}{r} \frac{\partial r \rho V \varepsilon}{\partial r}=\frac{1}{r} \frac{\partial}{\partial r}\left[r\left(\mu+\frac{v_{t}}{\sigma_{\varepsilon}}\right) \frac{\partial \varepsilon}{\partial r}\right]+C_{\varepsilon 1} \frac{\varepsilon}{k} P-C_{\varepsilon 2} \frac{\varepsilon^{2}}{k}
\end{gathered}
$$

in which $P$ is the generation rate of turbulence kinetic energy: $P=v_{t}\left[(\partial U / \partial r)^{2}+(\partial W / \partial r-W / r)^{2}\right]$, turbulence viscosity $v_{t}$ is modeled as $v_{t}=C_{\mu} k^{2} / \varepsilon$, and the constants are $C_{\mu}=0.09, \sigma_{k}=1.0, \sigma_{\varepsilon}=1.3$, $C_{\varepsilon 1}=1.45$ and $C_{\varepsilon 2}=1.90$. The Reynolds stresses appearing in Eqs. (2) and (3) are modeled as: $-\overline{u v}$ $=v_{t}(\partial U / \partial r)$ and $-\overline{v w}=v_{t}(\partial W / \partial r-W / r)$.

For the RS transport model, the Reynolds stress equations are in the form (EXPRESS)

Convection $\left({\overline{u_{i} u_{j}}}\right)=$ Diffusion $\left({\overline{u_{i} u_{j}}}\right)+$ Production $\left({\overline{u_{i} u_{j}}}\right)+$ Pressure-Strain $\overline{u_{i} u_{j}}$

- Dissipation $\left({\overline{u_{i}}}_{j}\right)$

where the convection and production terms are physically exact, the diffusion term is modeled by the Daly-Harlow gradient-transport model, and the pressure correlations are also modeled. They are

Convection: $\left({\overline{u_{i} u_{j}}}\right)=U_{k} \partial{\overline{u_{i} u_{j}}}_{j} / \partial x_{k}$

Production: $\left(\overline{u_{i} u_{j}}\right)=-\left(\overline{u_{i} u_{k}} \partial U_{j} / \partial x_{k}+\overline{u_{j} u_{k}} \partial U_{i} / \partial x_{k}\right)$

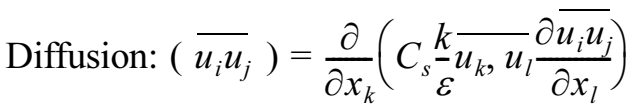

Pressure correlations: $\varphi_{i j, 1}=-C_{1}\left(\bar{u}_{i} u_{j}-1.5 \delta_{i j} P\right) \varepsilon / k, \varphi_{i j, 2}=-C_{2}\left(P_{i j}-1.5 \delta_{i j} P\right)$

The quantity $P_{i j}$ in Eq. $(7(\mathrm{e}))$ is a production component defined in Eq. $(7(\mathrm{c}))$. In practice, Eq. (7(a)) is simplified by neglecting tangential gradients due to axisymmetry and by neglecting many axial gradients (for instance, $\partial \bar{u}_{i}, u_{j} / \partial x_{k}$ ) according to the boundary layer theory. Eq. (6) is used to close the above equation form (7). The constants in the RS transport model are: $C_{1}=1.8, C_{2}=0.6, C_{s}=0.22$ and $C_{\varepsilon}=0.18$ (constants which are the same as the $k-\varepsilon$ model are no more listed). 


\subsection{Self-similarity of far-wake}

Self-similarity is assumed in the far-wake. Then the mean $U$-profile on a cross section at $x$ can be described as (Pope 2000)

$$
f(\xi)=\exp \left(-\xi^{2} \ln 2\right)=\frac{|U(x, R)-U(x, r)|}{U_{s}}
$$

where $\xi=r / r_{1 / 2}, r_{1 / 2}$ is the half radius, $R$ is the wake width, and $U_{s}=U(x, R)-U(x, 0)$. Fig. 1 shows the definitions of dimensions and velocities in self-similarity of the far-wake, in which $U_{0}$ is the wind speed from the upstream and $D$ is the diameter of the wind turbine. The half radius $r_{1 / 2}$ is defined as a position on the cross section where $U\left(x, r_{1 / 2}\right)=[U(x, R)+U(x, 0)] / 2$. Eq. (8(a)) is satisfied when $U(x, 0) / U(x, R)>0.9$ (Pope 2000).

To decide the wake width $R$, we assume $U(x, R)=99.9 \% U_{0}$ at the wake edge, $d r_{1 / 2} / d x$ is proportional to $d R / d x$, and use Eq. 8(b) as the following instead of Eq. (8(a)) above

$$
\exp \left(-\xi^{2} \ln 2\right)=\frac{|U-U(x, R)|}{U_{s}}=\frac{0.001 U_{0}}{0.999 U_{0}-U(x, 0)}
$$

With $U(x, 0)$ calculated in EXPRESS, we can solve $x$ from Eq. (8(b)), derive $r_{1 / 2}$ from the definition of $U\left(x, r_{1 / 2}\right)=\left[0.999 U_{0}+U(x, 0)\right] / 2$, and then obtain the wake width $R\left(=. \xi r_{1 / 2}\right)$ on this cross section. Thus, wake width expansion is predicted with the far-wake development.

\section{Numerical method}

A local radial coordinate is introduced, $h=r / R$. In the $x h$-coordinates, a general form of the basic equations (including Eqs. (1) to (7)) can be reduced to

$$
\frac{\partial \rho r \delta U \phi}{\partial x}+\frac{\partial r \rho(V-G U) \phi}{\partial \eta}=\frac{1}{r} \frac{\partial}{\partial \eta}\left(r \Gamma \frac{\partial \phi}{\partial r}\right)+r R S_{\phi}
$$

in which $G=d r / d x+\eta d R / d x, \phi$ represents an unknown variable of $U, V, r W$ (here $r W$ replaces $W$ ), $k, \varepsilon$ or a Reynolds stress, $\Gamma$ is the coefficient of the diffusion flux of $\phi$, and $S_{\phi}$ is the source of $\phi$.

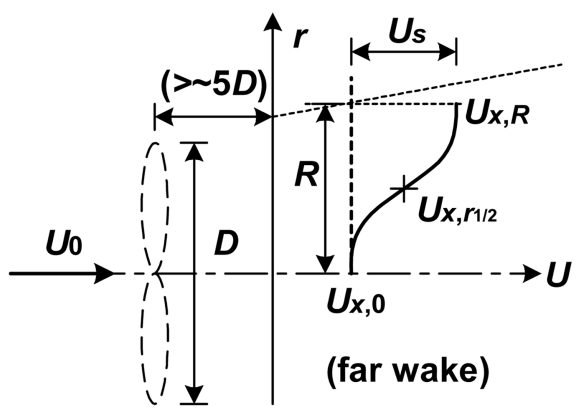

Fig. 1 Definitions of dimensions and velocities in the self-similarity assumption of the far-wake 
The introduction of the local coordinate, $\eta$, simplifies the solving process by the uniform equation form (9). In EXPRESS, computation is advanced cross section by cross section. Though the wake width grows with the cross section advancing, the normalized radial coordinate, $\eta$, remains between 0 and 1 . That's, the radial computational dimension is fixed from 0 to 1 at each cross section, which actually compresses the real wake dimension. Thus, the introduction of $\eta$ realizes the flow region marching by contracting (or expanding in other cases) computational grids. The finite volume approach is used to solve the governing equations. The scheme is hybrid with upwind- and central-differencing.

Boundary conditions are required at the axis and the wake edge because computation is advanced cross section by cross section. For the free and axisymmetric far-wake with the constant wind speed $U_{0}$, at the axis, $\partial U / \partial \eta=0, \partial p / \partial \eta=0$ (if needed), $V=W=0$, and shear stresses are zero; at the wake edge, $U=U_{0}, V=W=0, p=0$ (for convenience), and there are no shear stresses. The initial $U$ profile is defined in Eq. (8). For the far-wake with swirl, Burger's vortex model is used to set the initial $W$-profile (Susan-Resiga et al. 2006)

$$
W=\omega \frac{r_{0}^{2}}{r}\left(1-e^{-r^{2} / r_{0}^{2}}\right)
$$

where $\omega$ is a given angular velocity (varying from 0.5 to $51 / \mathrm{s}$ ), and the swirl core radius is $r_{0}=$ $0.2 D$. Initially, the tangential velocity and pressure are set to be zero.

We normalize velocities by $U_{0}$ (varying from 0.1 to $10 \mathrm{~m} / \mathrm{s}$ ), length dimensions by $D$ (fixed at 2.0 $\mathrm{m}$ ), and pressure by $\rho U_{0}^{2} / 2$ in results. Two dimensionless parameters are introduced, the Reynolds number and the swirl number (describing swirl strength (Hogg et al. 1989)), respectively

$$
R e=\frac{\rho U_{0} D}{\mu} \text { and } S n=\frac{\int_{0}^{\infty} U W r^{2} d r}{0.5 D \int_{0}^{\infty} U^{2} r d r}
$$

Similar computational boundary conditions are used when the far-wake is simulated in FLUENT with the $k-\varepsilon$ and RS transport models, respectively. Since it is not so easy to effectively mesh a domain with a large ratio of length to width in FLUENT as that in EXPRESS, a rectangular region of $1 D \times 12 D$ with the axis as a boundary is chosen and is meshed up to $320 \mathrm{~K}$ cells in total. In FLUENT, the condition of axial symmetry is used at the axis. At the upstream inlet, the velocity profile Eq. (8(a)) is set through UDF (user defined file). Pressure outlet (with zero velocity gradient) is applied upon the other two boundaries, the downstream outlet and the boundary away from the wake edge. The problem is steady and solved with the segregated solver in FLUENT. The basic equations are discretized with the second-order upwind scheme in space. PISO (Pressure-Implicit with Splitting of Operators) is used for the pressure-velocity coupling.

\section{Results and discussion}

\subsection{Verification of far-wake development model}

The initial $U$-profile defined in Eq. (8(a)) is used to verify the far-wake development model without swirl. According to the assumption of self-similarity, $r_{1 / 2} / D$ should be proportional to $(x / D)^{n}$ in the wake without swirl. Computation using EXPRESS shows the power $n$ is statistically a constant about 0.386 when $U(x, 0) / U(x, R) \geq 0.9$. Compared with the theoretical power of $1 / 3$ (Pope 
2000 ), the relative error is $16 \%$. One of the reasons that cause errors may be that the radial dimension in EXPRESS is limited, which differs from the radial infinity in theory. In the following discussion, $U(x, 0) / U(x, R)$ is set to be 0.9 for the initial $U$-profile.

\subsection{Wake width and half radius}

As expected, the wake width and the half radius increase with the wake developing in the axial direction (Fig. 2(a)), but the wake expands more in the radial direction at low Reynolds numbers than at high Reynolds numbers (Fig. 2(a)). At the same axial position, both wake width and half radius decrease with the Reynolds number (Fig. 2(b)). This indicates that high wind speed (usually with a high Reynolds number) may suppress wake expansion, compared with low wind speed. However, the suppressing effect of the high wind speed on the radial wake expansion is gradually weakened with the increment in the Reynolds number (Fig. 2(a)). Without swirl, the wake width curve takes 0.5 as the asymptote (Figs. 2(a) and 2(b)), which is the radius dimension of the wind turbine in the upstream. It can be expected that at a farther downstream cross section, a higher wind speed (with a higher Reynolds number) will be needed for wake width to reach 0.5 -asymptote. Fig. 2(a) also indicates that swirl may help the wake expand in the radial direction. With the swirl number at 0.17 , the asymptotic wake width is about 0.7 , which is $40 \%$ larger than that without swirl (Fig. 2(b)). The asymptotic half radius, 0.3, is also larger than that without swirl, 0.2 (Fig. 2(b)). Fig. 2(c) further shows that, at the same cross section, the radial dimension of the wake increases with the swirl strength.

\subsection{Velocity and pressure}

Without swirl in the wake, the lower Reynolds numbers (corresponding to the lower wind speed) result in more obvious increase of $U$ at the axis (Fig. 3(a)). With swirl, the axial velocity $U$ at the axis cannot increase so much as that without swirl (Fig. 3(a)). The maximum swirl velocity $W$ decreases with the axial growth of the wake (Fig. 3(a)). With viscous dissipation and the axial wake growth, both the $U$ - (with or without swirl) and $W$-profiles become flatter, as shown on the two cross sections at $x / D=0.025$ and 100 in Fig. 3(b). The radial velocity $V$ is insignificant in the farwake with or without swirl (Fig. 3(b)).
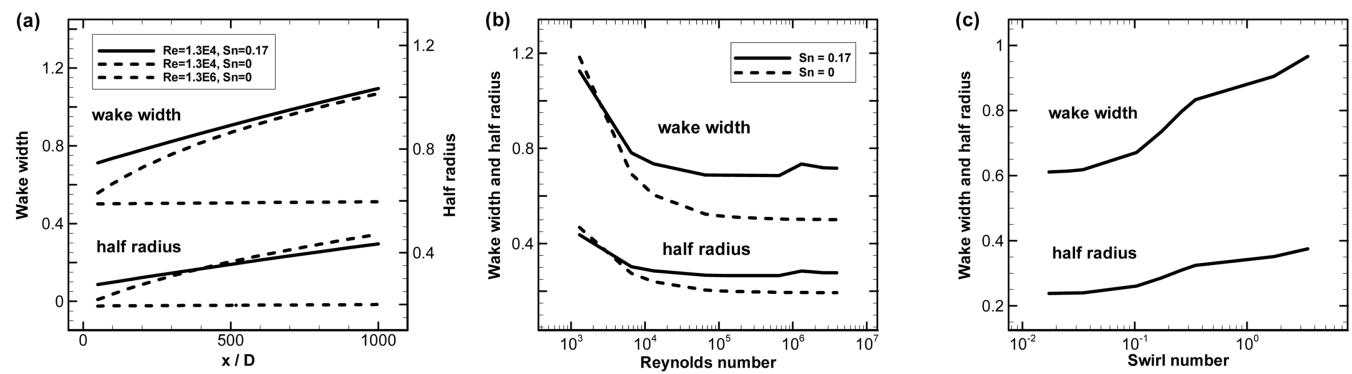

Fig. 2 The wake width and the half radius (a) as functions of $x / D$ at $R e=1.3 \times 10^{4}$ with $S n=0.17$, $R e=1.3 \times 10^{4}$ without swirl, and $R e=1.3 \times 10^{6}$ without swirl, (b) as functions of the Reynolds number at $S n=0$ and 0.17 , and $x / D=100$ and (c) as functions of the swirl number at $\operatorname{Re}=1.3 \times 10^{4}$ and $x / D=100$. 

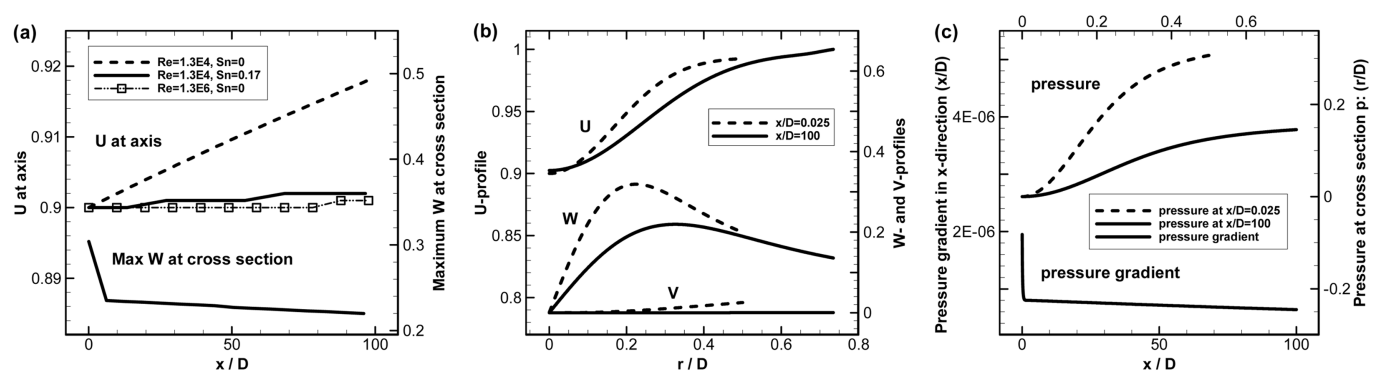

Fig. 3 (a) $U$ at the axis and maximum $W$ on cross sections as functions of $x / D$ at $R e=1.3 \times 10^{4}$ with $S n=0$ and 0.17 , and $R e=1.3 \times 10^{6}$ with $S n=0$, (b) the $U$-, $W$ - and $V$-profiles as functions of $r / D$ on two cross sections at $x / D=0.025$ and $100, R e=1.3 \times 10^{4}$, and $S n=0.17$ and (c) the axial gradient of pressure at the axis as a function of $x / D$ at $R e=1.3 \times 10^{4}$ and $S n=0.17$, and the pressure profile as a function of $r / D$ at $x / D=0.025$ and 100 .

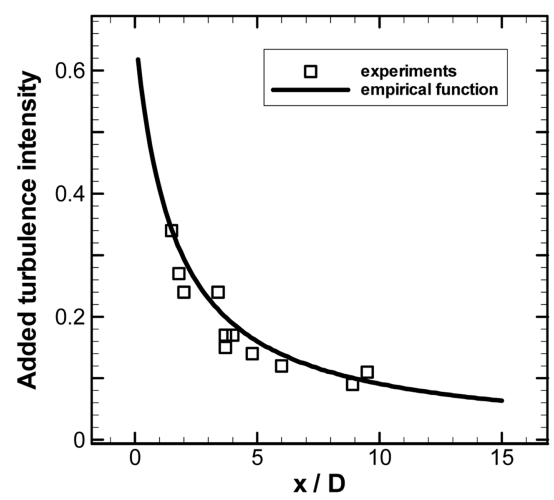

Fig. 4 The added turbulence intensity as a function of $x / D$ at $U(0,0)=9 \mathrm{~m} / \mathrm{s}$ and $R e=1.3 \times 10^{6}$. The experimental data are referred to the review of Vermeer et al. (2003)

For the free far-wake without swirl, the boundary layer theory neglects pressure variation. With swirl, the pressure increases with the radius on a cross section. This agrees with the pressure distribution of a vortex that the lowest pressure is at the vortex core. However, the positive and small axial gradient of pressure at the axis indicates that the pressure increases to a slight and slow degree (Fig. 3(c)). Generally pressure variation is not significant in the far-wake with swirl.

\subsection{Added turbulence intensity}

The added turbulence intensity in the far-wake is an important quantity that the Wind Energy Community is interested in. It is the additional turbulent kinetic energy created by a wind turbine, and should be added to the ambient one (Vermeer et al. 2003). One of the empirical functions is $I_{\text {add }}=[1.5+0.3(x / D) \sqrt{U(0,0)}]^{-1}(U(0,0)$ in $\mathrm{m} / \mathrm{s})$, which is regarded suitable for large $x$-values (Frandsen et al. 1999, Vermeer et al. 2003). The added turbulence intensity computed in EXPRESS shows good agreement with the experimental data, as shown in Fig. 4.

\subsection{Results comparison}

The two turbulence models in EXPRESS, $k-\varepsilon$ and RS transport, yield a similar development of 

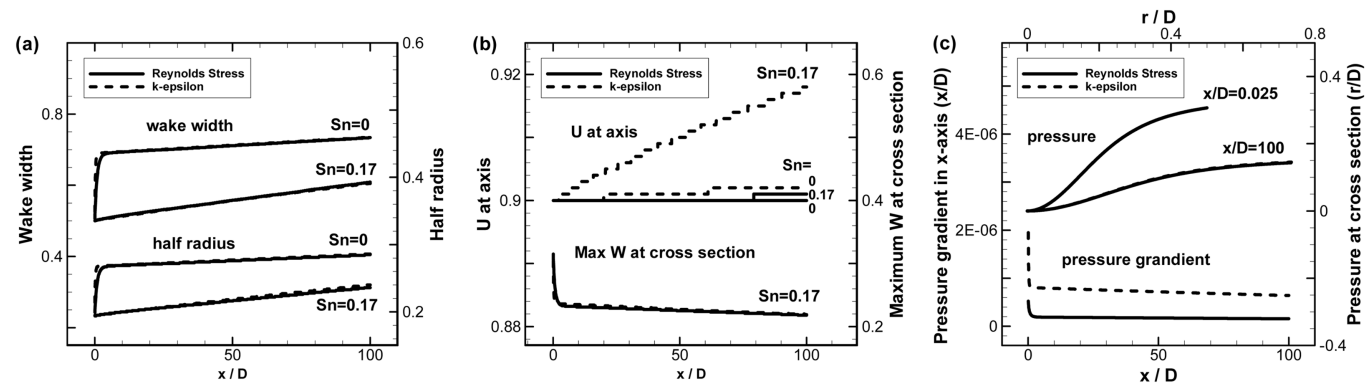

Fig. 5 Comparison of results from the $k-\varepsilon$ (dashed lines) and RS transport (solid lines) models at $R e=1.3 \times 10^{4}$. (a) The wake width and the half radius as functions of $x / D$ at $S n=0$ and 0.17 , (b) $U$ at the axis as a function of $x / D$ at $S n=0$ and 0.17 ; maximum $W$ at cross sections as a function of $x /$ $D$ at $S n=0.17$ and (c) the axial pressure gradient as a function of $x / D$ and the pressure profile on two cross sections at $x / D=0.025$ and 100 , and $S n=0.17$.

the wake width and the half radius (Fig. 5(a)). The axial velocity $U$ obviously increases at the axis only in the $k-\varepsilon$ model with swirl (Fig. 5(b)). The maximum swirl velocity $W$ on cross sections does not show difference between the two turbulence models (Fig. 5(b)). For pressure on cross sections, the two turbulence models result in the similar profiles (Fig. 5(c)). Although the $k$-e model shows the higher axial gradient of pressure than the RS transport model, the pressure gradient is generally so small as to be negligible (Fig. 5(c)).

It is generally regarded that the RS transport model is more accurate to simulate turbulence than the $k-\varepsilon$ model. Here, however, the two turbulence models show the similar results. In addition, it is noticed that the turbulent quantities, such as kinetic turbulent energy, dissipation rate, and Reynolds stresses, are generally pretty small, for example around $O\left(10^{-29}\right)$ (normalized by $\left.U_{0}^{2}\right)$, though their distributions on cross sections are still of self-similarity. One reason causing such disadvantages is that the boundary layer theory simplifies basic equations by neglecting some axial gradients and effects of the radial velocity. This affects accuracy of the RS transport model much more than that of the $k-\varepsilon$ model. The Reynolds stresses in the RS transport model are closely related to the velocity gradients in the axial direction and the radial velocity. In the $k-\varepsilon$ model, these Reynolds stresses are generally modeled. The assumptions of self-similarity and axisymmetry in the far-wake also introduce inaccuracy.

Further, the results are compared between the two computational tools, EXPRESS and FLUENT, still using the same turbulence models as above. The $U$ - and $W$-profile shapes from EXPRESS share more similarities with those from the RS transport model in FLUENT, than those from the $k-\varepsilon$ model in FLUENT (Figs. 6(a) and 6(b)). Since the RS transport model is more accurate than the $k-\varepsilon$ model to simulate turbulence in FLUENT, this comparison implies that the far-wake model in EXPRESS can ensure certain accuracy in wake velocity prediction. For either EXPRESS or FLUENT, the $k-\varepsilon$ and RS transport models result in little difference in the pressure profiles (Fig. 6(c)). However, the two computational tools yield different values. The $U$-profiles from FLUENT are below those from EXPRESS (Fig. 6(a)), whereas the $W$ - and pressure profiles show the contrary distributions (Figs. 6(b) and 6(c)). The maximum $w$ (Fig. 6(b)) and pressure (Fig. 6(c)) from either turbulence model in EXPRESS are about $75 \%$ and $50 \%$ of those from either turbulence model in FLUENT, respectively. The discrepancy between EXPRESS and FLUENT may be caused by the model based on the boundary layer theory, in which the Navier-Stokes equations are simplified and 

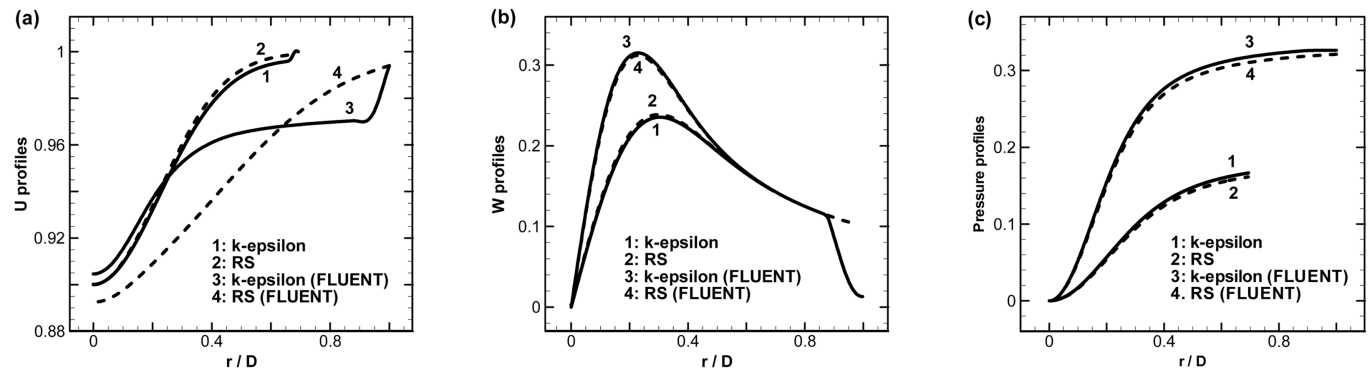

Fig. 6 (a) The $U$-, (b) $W$ - and (c) pressure profiles at $x / D=5, R e=1.3 \times 10^{4}$ and $S n=0.17$, using two turbulence models, $k-\varepsilon$ and RS transport, and two computational tools, EXPRESS and FLUENT.

the self-similarity assumption is made. Without an expanding grid, FLUENT gives profiles merely along the fixed width of the computational domain, while EXPRESS can show the expansion of the width. EXPRESS shows that the wake width is about $0.7 D$ at $x / D=5$ (Fig. 6).

\section{Conclusions}

Based on the boundary layer theory and self-similarity assumption, a model to study far-wake development behind a wind turbine is put forth. This model can predict far-wake development in large axial distances, and still allow small radial dimensions. The far-wake radially expands less at higher Reynolds numbers, while it expands more when swirl exists. The axial velocity and the swirl velocity (when swirl exists) are two dominant velocity components. The radial velocity is negligible. Swirl helps to accelerate the axial velocity at the axis. Pressure is insignificant in the farwake with or without swirl. The added turbulence intensity declines rapidly with the far-wake downstream development.

The model shows the following features: (i) The boundary layer theory simplifies the general Navier-Stokes equations, continuity equation, and turbulence model equations; (ii) simplicity of the basic equations compromises accuracy, and the RS transport model does not show obvious advantages over the $k-\varepsilon$ model; (iii) the numerical algorithm to solve the flow fields is simple, whereas very fine meshes are needed in the radial direction.

\section{Acknowledgements}

This research is supported by Wind Science \& Engineering Research Center, the Department of Civil Engineering, Texas Tech University, Lubbock, Texas, USA.

\section{References}

Crespo, A. and Hernandez, J. (1989), "Numerical modelling of the flow field in a wind turbine wake", Proceedings of the $3^{\text {rd }}$ Joint ASCE/ASME Mechanics Conference, ASME.

Crespo, A., Manuel, F., Moreno, D., Fraga, E. and Hernandez, J. (1985), "Numerical analysis of wind turbinewakes", Proceedings of the Delphi Workshop on Wind Energy Applications, Delphi. 
EXPRESS, A computer program for two-dimensional turbulent boundary layer flows, Imperial College, London.

Fletcher, T.M. and Brown, R.E. (2009), "Simulation wind turbine interactions using the vorticity transport model", Proceedings of the 47th AIAA Aerospace Sciences Meeting Including the New Horizons Forum and Aerospace Exposition, Orlando, Florida.

Frandsen, S. and Thogersen, M.L. (1999), "Integrated fatigue loading for wind turbines in wind farms by combining ambient turbulence and wakes", Wind Eng. Ind Aerod., 23(6), 327-340.

Hansen, M.O.L., Sorensen, J.N., Voutsinas, S., Sorensen, N. and Madsen, H.Aa. (2006), "State of the art in wind urbine aerodynamics and aeroelasticity", Prog. Aerosp. Sci., 42(4), 285-330.

Herbert, G.M.J., Iniyan, S., Sreevalsan, E. and Rajapandian, S. (2007), "A review of wind energy technologies", Renew. Sust. Energ. Rev., 11(6), 1117-1145.

Hogg, S. and Leschziner, M.A. (1989), "Computation of highly swirling confined flow with a Reynolds stress turbulence model”, AIAA J., 27(1), 57-63.

Katic, I., Hojstrup, J. and Jensen, N.O. (1986), "A Simple Model for Cluster Efficiency”, Proceedings of the EWEC'86.

Leschziner, M.A. and Rodi, W. (1984), "Computation of strongly swirling axisymmetryic free jets", AIAA J., 22(12), 1742-1747.

Lilley, D.G. (1973), "Prediction of inert turbulent swirl flows", AIAA J., 11(20), 955-960.

Lissaman P.B.S. (1979), "Energy effectiveness of arbitrary arrays of wind turbines", AIAA Paper, 79-0114, 1-7.

Mechali, M. and Barthelmie, R. (2006), "Wake effects at Horns Rev and their influence on energy production", EWEC 2006, 10.

Pope, S.B. (2000), Turbulent flows, Cambridge University Press.

Sanderse, B. (2009), "Aerodynamics of wind turbine wakes", Energy research Centre of the Netherlands, ECNE09-016.

Sumner, J., Watters, C.S. and Masson, C. (2010), "CFD in wind energy: the virtual, multiscale wind tunnel", Energies, 3(5), 989-1013.

Susan-Resiga, R., Ciocan, G.D., Anton, I. and Avellan, F. (2006), "Analysis of the swirling flow downstream a Francis turbine runner", J. Fluid Eng.-T. ASME, 128(1), 177-189.

Vermeer, L.J., Sorensen, J.N. and Crespo, A. (2003), "Wind turbine wake aerodynamics", Prog. Aerosp. Sci., 39(6-7), 467-510.

Vermeulen, P.E.J. (1980), “An experimental analysis of wind turbine wakes", Proceedings of the $3^{\text {rd }}$ International Symposium on Wind Energy Systems, BHRA Fluid Engineering, Lyngby, Denmark.

$C C$ 